\title{
Os distúrbios miofuncionais orofaciais na literatura odontológica: revisão crítica
}

\author{
Camila Cardoso Pereira*, Cláudia Maria de Felício**
}

\begin{abstract}
Resumo
Muitos estudos evidenciam a relação entre a forma e as funções do sistema estomatognático. Por esta razão, o objetivo do presente trabalho foi pesquisar em publicações da área Odontológica, em língua portuguesa, como são considerados os distúrbios miofuncionais orofaciais e, por conseqüência, como se dá a relação entre a Odontologia e a Fonoaudiologia. Para tanto, o método consistiu numa pesquisa bibliográfica em periódicos e livros veiculados na área odontológica, reunindo-se vinte e sete publicações do período de 1984 a 2002. Este levantamento teve como resultado o fato de que a maior parte dos autores relatam a necessidade de favorecer a condição miofuncional orofacial, visando a correção e a estabilidade do complexo orofacial. Quando odontólogos e fonoaudiólogos trabalham em equipe, o papel de cada profissional, bem como os conceitos envolvidos são apresentados com clareza. Entretanto, alguns autores relatam corrigir a má oclusão associada às alterações musculares e funcionais apenas com aparatos odontológicos. Ainda, há aqueles que não apresentam propostas para resolvê-los no plano de tratamento. Essa revisão nos levou à conclusão de que há diferenças nas condutas adotadas pelos odontológos no tratamento de seus pacientes, provavelmente decorrentes de suas formações, o que acarreta também posturas diferenciadas na interação com outros profissionais da saúde, dentre eles o fonoaudiólogo.
\end{abstract}

Palavras-chave: Odontologia. Fonoaudiologia. Comunicação interdisciplinar. Sistema estomatognático. Músculos faciais.

\section{INTRODUÇÃO}

Por meio de estudos desenvolvidos, fica cada vez mais evidente a necessidade de se considerar a estreita relação entre a condição óssea/dentária e a musculatura e funções do sistema estomatognático. Tem sido reconhecido que a oclusão modula as funções, mas também que não basta reposicionar as bases ósseas, e/ou adequar a oclusão dentária, se não for desenvolvida ou recuperada a funcionalidade para aquele "novo sistema", ou seja, se não for eliminada a existência de distúrbios musculares e funcionais que possam interferir de maneira

* Fonoaudióloga pós-graduada pela Universidade de Ribeirão Preto (UNAERP) - Nível Especialização. Mestre em Fonoaudiologia pela PUC/SP.

** Fonoaudióloga. Doutora em Ciências (Psicobiologia) pela Faculdade de Filosofia, Ciências e Letras de Ribeirão Preto da Universidade de São Paulo. Especialista em Motricidade Oral. Professora Doutora do Departamento de Oftalmologia, Otorrinolaringologia e Cirurgia de Cabeça e Pescoço da Faculdade de Medicina de Ribeirão Preto da Universidade de São Paulo. 
negativa no equilíbrio do sistema estomatognático. Isso já era reconhecido por Angle (1907 apud JANKELSON $\left.{ }^{1}, 1990\right)$ e vem sendo reafirmado continuamente $2,3,4,5,6,7,8,9,10,11,12,13$.

O fonoaudiólogo da área da Motricidade Oral é o "profissional responsável e competente no que se refere à avaliação, diagnóstico e tratamento das pessoas com distúrbios miofuncionais orofaciais e cervicais" (Documento Oficial do Comitê de Motricidade Oral, 02/2002 da Sociedade Brasileira de Fonoaudiologia-SBFa).

As primeiras especialidades da Odontologia a interagirem com a Fonoaudiologia foram a Odontopediatria e a Ortodontia. Posteriormente, outras como: Oclusão, Cirurgia Ortognática, Prótese e Periodontia passaram a considerar o papel da musculatura e funções como fatores etiológicos, perpetuantes ou agravantes de problemas antes considerados apenas de competência da Odontologia, que, portanto, passaram a ser também do escopo da Fonoaudiologia.

O número de equipes compostas por odontólogos e fonoaudiólogos cresceu consideravelmente nos últimos anos, e os resultados clínicos e de pesquisas se mostram promissores. Contudo, no cenário geral, de acordo com a opinião de diversos profissionais que atuam no âmbito clínico, ainda há dificuldade de comunicação e integração, pois muitos não consideram os benefícios da parceria.

Neste contexto, o objetivo do presente estudo foi realizar um levantamento bibliográfico de trabalhos veiculados na área odontológica, em língua portuguesa, visando verificar como são considerados os distúrbios miofuncionais orofaciais e, por conseqüência, como se dá a relação entre a Odontologia e a Fonoaudiologia.

\section{REVISÃO DE LITERATURA}

Souza e Muller de Araújo ${ }^{14}$ compararam os métodos mecânicos (placa com grade palatina), funcionais (placa reeducadora, visando a execução de exercícios reeducadores da língua) e mis- tos (placa reeducadora durante o dia e placa com grade palatina à noite), para o tratamento da deglutição atípica, e concluíram que o método misto mostrou-se mais eficiente.

Para Silva Filho et al. ${ }^{3}$, o sucesso do tratamento em casos de hábitos bucais deletérios depende do diagnóstico e planejamento realizados em equipe. Foi apresentado um caso de uma paciente de 5 anos com mordida aberta anterior, hábito de sucção ininterrupta do dedo polegar e interposição lingual secundária. Segundo os autores, a grade palatina fixa empregada funciona como um aparelho passivo impedidor de hábito, sendo que, a correção do desvio morfológico da oclusão depende da musculatura perioral. Posteriormente, foi instalada uma placa de contenção removível tipo Hawley, e a paciente foi encaminhada para avaliação fonoaudiológica, visando uma análise fonoarticulatória minuciosa.

Correia $^{15}$, com base na observação do índice significativo de pacientes com disfunção da articulação têmporo-mandibular que necessitavam de tratamento complementar na área fonoaudiológica, investigou a incidência de distúrbios fonoaudiológicos. Foram constatadas alterações nas funções de respiração (tipo), deglutição e fala; e a estrutura do sistema estomatognático que apresentou maior comprometimento foi a mandíbula, no que se refere aos seus movimentos, e o órgão fonoarticulatório mais comprometido foi a língua.

Segundo Okeson ${ }^{16}$, nas desordens têmporomandibulares (DTM) o tratamento definitivo é direcionado para alterar ou reduzir os fatores etiológicos. A terapia oclusal é realizada pelo cirurgião-dentista, e o psicólogo atua sobre o estresse emocional. Já terapia de suporte é direcionada aos sintomas. Dois tipos foram descritos, sendo uma para o alívio da dor (farmacológica e fisioterápica) e a outra para o alívio da disfunção (técnicas e exercícios que colaboram para ajudar a estabelecer uma função mandibular normal). 
Oliveira ${ }^{17}$ analisou a validade dos métodos fonéticos, aplicados em próteses para a determinação da dimensão vertical de oclusão, com enfoque multidisciplinar, em conjunto com a Fonoaudiologia e a Lingüística. A autora concluiu ser adequado o uso do fonema /s/, e que este método requer conhecimento de fonética para que se alcance bons resultados.

Cardoso et al. ${ }^{5}$ relataram um caso clínico de uma paciente de 28 anos, apresentando problema estético, dentre outras coisas com intrusão do elemento 21, relacionada à sucção de língua e à fonação atípica. $\mathrm{O}$ tratamento incluiu especialidades, tais como Fonoaudiologia, Oclusão, Ortodontia, Periodontia, Dentística e Prótese, a fim de que fossem atendidas as necessidades da paciente. Os autores concluíram que o dentista tem uma grande responsabilidade para com o paciente que o procura, e deve organizar a ação cooperativa de um plano de tratamento com abordagem multidisciplinar.

De acordo com Correia ${ }^{18}$, o adequado funcionamento da ATM depende da harmonia entre a neuromusculatura, a articulação propriamente dita e a oclusão dentária. Dentre os pacientes acometidos pela desordem da ATM, 62,9\% apresentavam algum tipo de alteração nos órgãos fonoarticulatórios - lábios, língua e bochechas e nas funções de deglutição, mastigação, respiração e fala, necessitando da atuação fonoaudiológica para sua completa reabilitação.

Andrade e Justiniano ${ }^{6}$ apresentaram casos clínicos de mordida aberta, tratados com a aparatologia Ortopédica Maxilar. Esclareceram que, devido à diversidade etiológica, todos os pacientes com mordida aberta, de qualquer natureza, são rotineiramente avaliados e tratados pelo ortopedista funcional, otorrinolaringologista e fonoaudiólogo. Com isso, têm evitado as recidivas que comumente ocorrem em terapias menos abrangentes.

Farret et al. ${ }^{19}$ investigaram os resultados do tratamento da deglutição atípica em 20 crianças, entre 8 e 12 anos, que foram divididas aleatoriamente em dois grupos (GA - tratamento mecânico com mioterapia; GB - apenas tratamento mecânico). Cada indivíduo recebeu a placa reeducadora (uso diurno) e a impedidora (uso noturno). A correção do padrão de deglutição ocorreu em $60 \%$ dos indivíduos do GA e $20 \%$ dos indivíduos do GB. Os autores concluíram que o uso isolado de placas é ineficiente, e os resultados se mostraram mais favoráveis com a associação da mioterapia.

Farret et al. ${ }^{20}$ avaliaram o índice de recidiva de deglutição atípica após 3 anos a contar do final do tratamento, comparando crianças submetidas à terapia miofuncional associada à mecânica (GA) e apenas ao tratamento mecânico (GB). Concluíram que a associação da terapia miofuncional à mecânica favoreceu as condições para mudanças da deglutição e da posição de repouso.

Moraes et al. ${ }^{7}$ empregaram Ortodontia, implante e terapia miofuncional em um caso de tratamento corretivo dentofacial com ausência de dentes. Os autores se pautaram na idéia de que os implantes osseointegrados estão sujeitos a uma série de fatores, dentre eles o "ambiente muscular" (lábios, língua e bochechas). Assim, a terapia miofuncional, além da normalização da neuro-musculatura, permite uma melhor estabilidade para o tratamento corretivo ortodôntico e para a fisiologia do complexo estomatognático-facial como um todo.

Almeida et al. ${ }^{21}$ explicaram que, com relação à etiologia da mordida aberta anterior, qualquer interferência no período de crescimento ativo das estruturas da face, pode alterar a morfologia e a função do sistema estomatognático, prejudicando o desenvolvimento oclusal e esquelético normal. Quanto ao tratamento, os autores acreditam que a abordagem ideal deva ser de caráter multidisciplinar - odontológo, fonoaudiólogo, otorrinolaringologista e psicólogo.

Mongini $^{22}$ escreveu a respeito do tratamento 
fisioterápico e de exercícios para a musculatura nos casos de DTM. Dentre as técnicas citou: a eletroestimulação transcutânea (TENS), o soft-laser, a aplicação de calor ou frio e as injeções anestésicas. Com relação aos exercícios para os músculos elevadores e a ATM, recomendou movimentos de abertura e fechamento da boca, estabilização rítmica, exercício de contra-resistência e de tração articular.

Silva Filho et al. ${ }^{23}$ relataram a reabilitação facial e oclusal de um paciente portador de fissura unilateral completa de lábio e palato, por meio de Ortodontia, cirurgia ortognática e cirurgia plástica. Entretanto, esclareceram que neste trabalho foi citado apenas um dos pilares do longo processo reabilitador do paciente fissurado que deve sempre incluir, entre outras especialidades, a Fonoaudiologia, para que a reabilitação seja completa e consiga reintegrar o indivíduo no seu universo social.

Comin e Passos Filho ${ }^{24}$ apontaram que há dúvidas quanto à indicação de tratamento fonoaudiológico para o restabelecimento das funções orais em pacientes que utilizam aparelhos ortopédicos funcionais. Baseados na literatura, concluíram que a terapia miofuncional realizada pelo fonoaudiólogo não é substitutiva dos aparelhos funcionais, tão pouco os aparelhos por si só podem assegurar resultados satisfatórios, sendo necessária a integração de áreas para a normalização morfológica e funcional. Acrescentaram, que a avaliação, o diagnóstico funcional e a indicação da terapia miofuncional competem ao fonoaudiólogo.

Felício e Mazzetto ${ }^{10}$ apontaram as metas terapêuticas e sugestões de condutas para os casos de DTM, que de modo geral visam favorecer o equilíbrio do sistema estomatognático, propiciar uma harmonia côndilo-disco e minimizar a dor. Esclareceram que há condutas diferenciadas e específicas, devendo existir uma real correspondência entre as metas e procedimentos realizados pelo cirurgião-dentista e o fonoaudiólogo.

Lino $^{25}$ escreveu que em casos de deglutição atípica o fonoaudiólogo tráz grande auxílio com o emprego de métodos reeducativos, porém, o ortodontista e o odontopediatra devem estabelecer uma terapêutica clínica imediata. Por melhor que sejam os resultados obtidos com outros métodos, todos os pacientes devem ser submetidos a tratamento reeducativo, pois sem isso, após remoção do dispositivo, a deglutição atípica poderá recidivar. Para tanto, propôs o uso da placa reeducadora e a realização de exercícios.

Rakosi et al. ${ }^{26}$ realizaram um estudo acerca de tratamentos ortodônticos e ortopédicos. Nos três casos apresentados, foram constatadas disfunções orofaciais. O primeiro caso apresentava deglutição atípica e sucção labial; o segundo respiração bucal, deglutição atípica, onicofagia e sigmatismo interdental; e o terceiro sucção digital de longa duração, disfunção lingual (função e repouso), bem como sinais e sintomas de DTM. De acordo com o plano de tratamento proposto, apenas para o primeiro caso foi sugerida terapia miofuncional, se a interposição lingual persistisse após a correção morfológica.

Tukasan et al. ${ }^{27}$ descreveram um caso clínico de mordida aberta anterior, devido ao hábito de sucção digital, associado à mordida cruzada posterior unilateral, na fase de dentição mista. A paciente apresentava deglutição atípica, problemas rinofaríngeos, respiração do tipo mista e dificuldades em alguns fonemas. Foi realizada a correção da má oclusão e indicado um aparelho removível com escudo palatino de resina acrílica, para controle do hábito de sucção digital e da postura de língua, possibilitando a correção da mordida aberta.

Segundo Henriques et al. ${ }^{11}$, para que o tratamento ortodôntico da mordida aberta anterior seja efetivo e estável, uma abordagem multidisciplinar, incluindo a Psicologia, a Otorrinolaringologia e a Fonoaudiologia é necessária, pois não basta apenas a correção do problema morfológico. A conduta terapêutica a ser seguida está diretamente relacionada com a etiologia e com a época de intervenção.

Ritter $^{28}$ relatou um caso clínico de um paciente 
de 17 anos, com fonação e deglutição atípica, portador de uma deformidade dentofacial, com má oclusão de Classe II, divisão 1, e mordida aberta anterior, apresentando também ausência do selamento labial e excesso de exposição dos incisivos superiores. O tratamento realizado envolveu Ortodontia e cirurgia ortognática.

Segundo Zarb et al. ${ }^{29}$, o primeiro objetivo do tratamento das DTMs é o controle da dor e do desconforto, obtidos através de tratamento medicamentoso e de exercícios, visando obter o relaxamento muscular; treinar uma função muscular rítmica e coordenada; aumentar a amplitude do movimento mandibular e a força muscular.

Borges et al. ${ }^{30}$ abordaram o tratamento da deglutição atípica com pressionamento lingual. Dos onze textos revisados, seis indicavam o tratamento ortodôntico associado à terapia miofuncional, realizada por fonoaudiólogos, afirmando que esta interação é essencial para o sucesso e estabilidade do tratamento, eliminando os riscos de recidivas. Em contrapartida, cinco referências priorizavam a mecanoterapia.

Segundo Cozzani ${ }^{31}$, a postura e os hábitos incorretos podem levar a alterações na posição dos dentes e no esqueleto, além de recidiva após o tratamento, se não corrigidos. $\mathrm{O}$ autor citou um caso clínico de uma paciente de 17 anos de idade, tendo como diagnóstico Classe III e mordida aberta anterior, com pressão lingual persistente entre os arcos dentários. $\mathrm{O}$ plano terapêutico empregado foi aparelho fixo e uso de elásticos anteriores. Um resultado razoável foi obtido, em termos da correção da Classe III. No entanto, ocorreu recidiva da mordida aberta anterior, após onze anos do término do tratamento ortodôntico.

Kasai e Portella ${ }^{32}$ discutiram sobre a importância do tratamento fonoaudiológico do paciente ortodôntico-cirúrgico, pois as correções ósseas e dentárias ocorrerão, exigindo adaptação das funções a esta nova condição. Para as autoras, o equilíbrio será conseguido através do tratamento ortodôntico-cirúrgico e também da correção das funções do sistema estomatognático.

Kuramae et al. ${ }^{33}$ classificaram a deglutição atípica com interposição lingual em alta, média e baixa. Para o tratamento da deglutição atípica com interposição alta e média utilizam placas reeducadoras-impedidoras convencionais, na arcada superior. Os casos com postura lingual baixa ou mesmo média, são tratados com placa reeducadora-impedidora inferiores (placa geniana). Acrescentaram que, associam exercícios mioterápicos para reeducar a postura da língua, visando o restabelecimento do padrão normal da deglutição.

Kuramae et al. ${ }^{34}$ sugeriram que para o tratamento da deglutição atípica, o método misto, associando o método mecânico (realizado por ortodontistas, através do uso de aparelhos, seja para impedir que a língua se projete entre os dentes, seja para guiar a língua para uma posição correta) e os métodos funcionais (executados por fonoaudiólogos) é o mais efetivo, devendo haver uma competente interação entre a Fonoaudiologia e a Ortodontia.

De acordo com Mercadante ${ }^{35}$, no que se refere ao plano de tratamento da deglutição atípica, deve-se associar exercícios mioterápicos realizados por fonoaudiólogos, com métodos mecânicos, executados por ortodontistas. Para os casos de respiração bucal sugeriu o encaminhamento ao otorrinolaringologista, e, ainda, que a musculatura seja reabilitada por meio de exercícios funcionais.

Silva Filho et al. ${ }^{4}$ discutiram os efeitos induzidos pelo uso da grade palatina fixa nos estágios de dentaduras decídua e mista, sobre a mordida aberta anterior, e a necessidade de fortalecimento da musculatura peribucal, com exercícios programados e orientados pelo fonoaudiólogo. Os autores são adeptos da grade palatina fixa, seguida simultaneamente com a atuação fonoaudiológica, que potencializa o processo corretivo da má oclusão, 
ao valorizar a tonicidade da musculatura peribucal.

Kuramae et al. ${ }^{36}$ descreveram um caso clínico de uma paciente de 10 anos que apresentava deglutição atípica do tipo baixa e má oclusão de Classe II, divisão 1, acompanhada de mordida aberta anterior e mordida cruzada posterior unilateral. Para o tratamento interceptador, instalaram um aparelho ortodôntico removível, a placa geniana de Nouer - visando a correção do hábito de deglutição atípica com interposição lingual baixa e da mordida aberta anterior. Após a irrupção dos dentes permanentes, a paciente foi encaminhada para o tratamento ortodôntico corretivo.

Bortolozo et al. ${ }^{13}$ apresentaram um caso clínico acerca da expansão rápida e tração maxilar e concluíram que o tratamento realizado com acompanhamento fonoaudiológico devolve ao paciente uma relação oclusal, estética e funcional mais favorável, possibilitando ainda que o equilíbrio forma-função seja mantido durante o crescimento.

Melgaço et al. ${ }^{37}$ enfatizaram a importância da integração de médicos, odontológos e fonoaudiológos nos casos de fissuras labiopalatinas. Esclareceram que o ortodontista atua na correção dos desvios do crescimento facial e dos distúrbios oclusais e o fonoaudiólogo restabelece as funções estomatognáticas (fala, sucção, mastigação, deglutição e respiração) e atua nas áreas de linguagem, voz e audição, quando alteradas. Estando estas áreas intimamente relacionadas, os profissionais devem buscar um atendimento globalizado e eficiente, com o intuito de proporcionar uma melhor qualidade de vida ao paciente.

\section{DISCUSSÃO}

De posse deste levantamento bibliográfico, é importante fazer uma síntese das opiniões dos autores no que se refere aos distúrbios miofuncionais orofaciais e ao trabalho desenvolvido pelo fonoaudiólogo.

Dentre os autores pesquisados, verificou-se que a musculatura e funções estomatognáticas como fator de equilíbrio ou disfunção do sistema estomatognático, de acordo com a condição fisiológica, foi destacada pela maioria dos autores $3,4,5,6,7,10,11,13,14,15,17,18,19,20,21,23,24,32,33,34,35,36,37$.

Vários deles mencionaram a importância da integração da Odontologia e da Fonoaudiologia no tratamento do complexo orofacial, enfatizando que a terapia miofuncional trabalha não somente para a normalização da neuromusculatura, como também permite o maior equilíbrio do sistema estomatognático, estabilidade e sucesso quanto aos resultados $3,4,5,6,7,10,11,13,19,20,21,23$, 24,30,32,34,35, 37 .

Assim, o ortodontista deve possuir uma visão global do tratamento, para que seus encaminhamentos sejam mais precisos, culminando numa terapia eficaz e diminuindo o risco de recidiva da má oclusão ${ }^{11}$.

Além da Ortodontia, outras especialidades consideram importante a participação do fonoaudiólogo no planejamento e/ou no tratamento, dentre elas a reabilitação oral ${ }^{17}$, a Odontologia estética ${ }^{5}$ e a Implantologia ${ }^{7}$.

Em contrapartida, alguns autores, apesar de citarem a importância da neuromusculatura como fator desencadeante ou perpetuante de alterações morfológicas, propõem métodos mecânicos, juntamente com exercícios, realizados a partir de placa reeducadora ou outro dispositivo intrabucal, prescritos e orientados pelo dentis$\operatorname{ta}^{14,25,31,33,36}$.

Contudo, Lino ${ }^{25}$ mencionou a importância da intervenção fonoaudiológica para o sucesso da adequação da deglutição e demonstrou uma visão abrangente sobre o sistema estomatognático, considerando a necessidade de reeducação da musculatura, o que merece destaque, principalmente pela época em que foi escrito originalmente o capítulo (aqui citada a reedição).

Alguns autores, também, destacaram que os distúrbios miofuncionais são considerados fatores etiológicos de má oclusão ${ }^{26,27,28}$, mas não fizeram menção de estra- 
tégias para a intervenção.

Com relação às disfunções têmporo-mandibulares, estudos comprovam a prevalência de alterações miofuncionais ${ }^{15,18}$. Isso indica que é indispensável a inter-relação de áreas, para um prognóstico favorável, sendo necessário e possível favorecer o equilíbrio do sistema estomatognático por meio de terapia em motricidade orofacial $^{10}$, focalizando e trabalhando as funções - mastigação, deglutição, respiração e fala - o que no Brasil é realizado por fonoaudiólogos.

Contudo, há quem considere a disfunção têmporo-mandibular somente sob a ótica odontológica, ou incluem a psicológica, enquanto outras modalidades terapêuticas são consideradas apenas terapias de suporte, apesar de ter sido comentada a importância da integridade das estruturas e funções do complexo orofacial na determinação da estabilidade dentária e funcional e exercícios sugeridos $^{16,22,29}$.

Observou-se que, quando odontólogos e fonoaudiólogos trabalham em equipe, o que pode ser identificado pela autoria dos textos, os conceitos relativos à musculatura, às funções orofaciais e aos distúrbios miofuncionais orofaciais são expressos de modo mais preciso e coerente, sendo também estabelecido com clareza o papel de cada profissional.

\section{CONCLUSÃO}

Em todas as 32 publicações constatou-se que a relação "forma-função" foi focalizada pelos autores. Alguns consideram necessário o tratamento fonoaudiológico associado ao odontológico para a adequação e estabilidade do complexo orofacial, outros empregam aparelhos ortodônticos/ortopédicos, visando a adequação tanto morfológica como funcional. Ainda, há aqueles que apenas mencionam os distúrbios miofuncionais orofaciais como fatores etiológicos e/ou agravantes da má oclusão, mas não apresentam propostas para resolvê-los no plano de tratamento.
Essa revisão, portanto, evidencia que há diferenças nas condutas adotadas pelos odontológos no tratamento de seus pacientes, provavelmente decorrente de suas formações, o que pode acarretar também posturas diferenciadas na interação com outros profissionais da saúde, dentre eles o fonoaudiólogo. As diferenças observadas não estão diretamente relacionadas à época em que foram escritos os textos.

O diálogo entre odontológos e fonoaudiólogos poderia contribuir para a melhor compreensão dos casos clínicos nos quais se associam distúrbios oclusais e miofuncionais, assim como para a definição das metas e condutas a serem aplicadas no tratamento. 


\title{
The orofacials myofunctional disorders in the dentistry publication: critical review
}

\begin{abstract}
Many studies evidence the relation between the form and the functions of the stomatognathic system. For this reason, the objective of the present work is to search through the Dentistry Publication, in portuguese language, how the Orofacial Myofunctional Disorders are considered, and, as a result, how the relation between the Dentistry and Speech Therapy works. For this, the method consisted of a bibliographical research in journals and books of the Dentistry area, congregating twenty and seven publications from 1984 through 2002. As a result, the survey showed the fact that most of the authors report the needs of favoring the orofacial myofunctional condition, aiming at the correction and the stability of the orofacial complex. When Dentists and Speech therapists work together, the role of each professional, as well as the involved concepts are clearly presented. However, some authors report correcting the bad occlusion associated with the muscular and functional alterations only with dentistry apparatuses. Yet, there are some dentists that do not present any proposals to solve it in the treatment plan. This review lead to the conclusion that there are differences in the behaviors adopted by the dentists in the treatment of their patients, probably resulting of its formations, what also causes differentiated positions the interaction with other health professionals, as the Speech therapists.
\end{abstract}

Key words: Dentistry. Speech therapy. Interdisciplinary communication. Stomatognathic system. Facial muscles.

\section{REFERÊNCIAS}

1. JANKELSON, R. R. Neuromuscular dental diagnosis and treatment. St. Louis: Ishiyaku Euroamericana, 1990.

2. PADOVAN, B. A. E. Correlação entre a odontologia e fonoaudiologia. J Bras Ortodon Ortop Facial, Curitiba, v. 1, n. 2, p. 34-37, mar./abr. 1996.

3. SILVA FILHO, O. G. da; OKADA, T.; SANTOS, S. D. dos. Sucção digital: abordagem multidisciplinar: ortodontia X psicologia $X$ fonoaudiologia. Estomatol Cult, Baurú, v.16, n. 2, p. 44-53, 1986.

5. CARDOSO, A. C.; CANTO, G. L.; DERECH, C. D'A. Odontologia estética: uma necessidade multidisciplinar. Rev Bras Odontol, Rio de Janeiro, 52, n. 2, p. 47-49, 1995.

6. ANDRADE, L. L.; JUSTINIANO R. Tratamento da síndrome de respiração bucal com mordida aberta através da aparatologia ortopédica funcional. J Bras Ortodon Ortop Facial, Curitiba, v. 1, n. 2, p. 3-13, mar./abr. 1996.

7. MORAES, S. H.; KÖHLER, G. I.; KÖHLER J. F. W.; KÖHLER N. R. W.; KÖHLER V. L. B. Tratamento combinado ortodontia-implante-mioterapia: relato de um caso clínico. J Bras Odontol Clín, Curitiba, n. 1, n. 5, p. 21-24, set./out. 1997.

8. MARCHESAN, I. Q. Fundamentos em fonoaudiologia: aspectos clínicos da motricidade oral. Rio de Janeiro: Guanabara, 1998.

9. FELÍCIO, C. M. Fonoaudiologia nas desordens temporomandibulares: uma ação educativa: terapêutica. São Paulo: Pancast, 1994.

10. FELÍCIO, C. M.; MAZZETTO, M. O. Desordens musculares e intra-articulares: características, metas e condutas terapêuticas. In: FELíCIO, C. M. de. Fonoaudiologia aplicada a casos odontológicos: motricidade oral e audiologia. São Paulo: Pancast, 1999.

11. HENRIQUES, J. F. C.; JANSON, G.; ALMEIDA, R. R.; DAINESI, E. A.; HAYASAKI, S. M. Mordida aberta anterior: a importância da abordagem multidisciplinar e considerações sobre etiologia, diagnóstico e tratamento. Apresentação de um caso clínico. R Dental Press Ortodon Ortop Facial, Maringá, v. 5, n. 3, p. 29-36, maio/jun. 2000.
12. BIANCHINI, E. M. G. Avaliação fonoaudiológica da motricidade oral: distúrbios miofuncionais orofaciais ou situações adaptativas. R Dental Press Ortodon Ortop Facial, Maringá, v. 6, n. 3, p. 73-82, maio/jun. 2001.

13. BORTOLOZO, M. A.; TOMÉ, M. C.; KRÜGER, A. R. Terapia interdisciplinar: expansão rápida e tração maxilar com acompanhamento fonoaudiológico. R Dental Press Ortodon Ortop Facial, Maringá, v. 6, n. 7, p. 69-79, 2002.

14. SOUZA, D. M. K.; MULLER DE ARAÚJO, M. C. Avaliação clínica dos métodos terapêuticos da deglutição atípica. RGO, Porto Alegre, v. 32, n. 3, p. 183-188, jul./set. 1984.

15. CORREIA, F. A. S. Disfunção da articulação temporomandibular e seu relacionamento com distúrbios fonoaudiológicos: contribuição ao estudo. 1988. Tese (Doutorado)-Faculdade de Odontologia, Universidade de São Paulo, São Paulo, 1988.

16. OKESON, J. P. Fundamentos de oclusão e desordens temporo-mandibulares. 2. ed. São Paulo: Artes Médicas, 1992.

17. OLIVEIRA, M. A. P. Estudo da validade dos métodos fonéticos para determinação da dimensão vertical em prótese. 1994. Tese (Doutorado)- Faculdade de Odontologia, Universidade de São Paulo, São Paulo, 1994.

18. CORREIA, F. A. S. Aspectos fonoaudiológicos da ATM. In: BARROS, J. J.; RODE, S. M. Tratamento das disfunções craniomandibulares: ATM. São Paulo: Ed. Santos, 1995.

19. FARRET, M. M. B.; TOMÉ, M. C.; JURACH, E. M.; MARCHIORI, S. C. Proposta de um tratamento para reposicionamento lingual em pacientes portadores de deglutição atípica. Ortodontia, São Paulo, v. 29, n.1, p. 43-47, jan./abr. 1996.

20. FARRET, M. M. B.; JURACH, E. M.; TOMÉ, M. C. Análise do comportamento da deglutição em crianças submetidas a tratamento mioterápico associado ao uso de placas reeducadoras e impedidoras. R Dental Press Ortodon Ortop Facial, Maringá, v. 2, n. 5, p. 91-95, set./out. 1997.

21. ALMEIDA, R. R. de.; SANTOS, S. C. B. N.; SANTOS, E. C. A.; INSABRALDE, C. M. B.; ALMEIDA, M. R. Mordida aberta anterior: considerações e apresentação de um caso clínico. R Dental Press Ortodon Ortop Facial, Maringá, v. 3, n. 2, p. 17-29, mar./ abr. 1998. 
22. MONGINI, F. ATM e músculos craniocervicofaciais: fisiopatologia e tratamento. São Paulo: Ed. Santos, 1998.

23. SILVA FILHO, O. G. da; FERRARI JÚNIOR, F. M.; CARVALHO, R. M.; MAZZOTINI, R. A cirurgia ortognática na reabilitação do paciente portador de fissura unilateral completa de lábio e palato. R Dental Press Ortodon Ortop Facial, Maringá, v. 3, n. 4 , p. 51-70, jul./ago. 1998

24. COMIN, I.; PASSOS FILHO, L. P. Ortopedia funcional dos maxilares e fonoaudiologia: uma possibilidade terapêutica multidisciplinar. R Dental Press Ortodon Ortop Facial , Maringá, v. 4, n.4, p. 63-70, jul./ago. 1999.

25. LINO, A. P. Introdução ao problema da deglutição atípica. In: INTERLANDI, S. Ortodontia: bases para a iniciação. São Paulo: Artes Médicas, 1999.

26. RAKOSI, T.; JONAS, I.; GRABER, T. M. Ortodontia e ortopedia facial: diagnóstico. Porto Alegre: Artmed, 1999.

27. TUKASAN, P. C.; ALMEIDA, I. A. de; MAGNANI, M. B. B. A.; ALMEIDA, M. H. C. de. Mordida aberta anterior associada à mordida cruzada posterior: relato de um caso clínico. J Bras Ortodon Ortop Facial, Curitiba, v. 5, n. 4, 1999.

28. RITTER, D. E. Tratamento orto-cirúrgico em pacientes portador de classe II, divisão $1 \mathrm{com}$ mutilação e mordida aberta anterior. R Dental Press Ortodon Ortop Facial, Maringá, v. 5, n. 1, p. $45-50$, jan./fev. 2000

29. ZARB, A. G.; CARLSSON, E.G. Controle clínico. In: ZARB, A. G.; CARLSSON, E. G.; SESSLE, J. B.; MOHL, D. N. Disfunções da articulação temporomandibular e dos músculos da mastigação. São Paulo: Ed. Santos, 2000.

30. BORGES, C. S.; VEDOVELLO, F. M.; VALDRIGUI, H. C. Conside- rações sobre o diagnóstico e o tratamento da deglutição atípica com pressionamento anormal da língua. Ortodontia, São Pauo, v. 34, n. 3, p. 74-79, set./dez. 2001

31. COZZANI, G. O jardim da ortodontia: reflexões, sugestões e erros. São Paulo: Quintessence, 2001.

32. KASAI, R. C. B.; PORTELLA, M. Q. Intervenção fonoaudiológica em pacientes submetidos ao tratamento ortodôntico-cirúrgico. R Dental Press Ortodon Ortop Facial, Maringá, v. 6, n. 2 p. 79-84, mar./abr. 2001

33. KURAMAE, M.; NOÜER, D. F.; ALMEIDA, M. H. C.de; MAGNA $\mathrm{NI}, \mathrm{M}$. B. B. A. Uma proposta de classificação para a deglutição atípica com interposição lingual. J Bras Ortodon Ortop Facial Curitiba, v. 6, n. 33, p. 205-212, maio/jun. 2001.

34. KURAMAE, M.; TAVARES, S. W.; NOÜER, D. F.; MAGNANI, M. B. B. A. Deglutição atípica com interposição lingual: etiologia classificação, diagnóstico e terapêutica. Rev Odontol UNICID São Paulo, v. 13, n. 3, p. 221-230, set./dez. 2001.

35. MERCADANTE, M. M. N. Hábitos em ortodontia. In: FERREIRA, Flávio Velini. Ortodontia: diagnóstico e planejamento clínico. São Paulo: Artes Médicas, 2001.

36. KURAMAE, M.: TAVARES, S. W.: ALMEIDA, H. A. de; ALMEIDA, M. H. C. de; NOUER, D. F. Correção da deglutição atípica associada à mordida aberta anterior: relato de caso clínico. J Bras Ortodon Ortop Facial, Curitiba, v. 6, n. 36, p. 493-501, nov./dez. 2001

37. MELGAÇO, C. A.; DI NINNO, C. Q. M. S.; PENNA, L. M.; VALE, M. P. P. Aspectos ortodônticos/ortopédicos e fonoaudiológicos relacionados a pacientes portadores de fissuras labiopalatinas. J Bras Ortodon Ortop Facial, Curitiba, v. 7, n. 37, p. 23-32, jan./fev. 2002.
Endereço para correspondência

Camila Cardoso Pereira

Rua Manoel Inácio de Souza, 24. Apt. 403, Jd. dos Estados CEP: 79020-220

Campo Grande/MS

E-mail: camilacardoso@terra.com.br 